\title{
A Linguistic-Valued Approximate Reasoning Approach for Financial Decision Making
}

\author{
Xin Liu ${ }^{1}$, Ying Wang ${ }^{2}$, Xiaonan $\mathrm{Li}^{2}$, and $\mathrm{Li} \mathrm{Zou}^{2 *}$ \\ ${ }^{1}$ School of Mathematics, Liaoning Normal University, \\ Dalian 116029, China \\ E-mail:liuxin67812@163.com \\ ${ }^{2}$ School of Computer and Information Technology, Liaoning Normal University, \\ Dalian 116081, China \\ *E-mail: zoulicn@163.com
}

Received 11 April 2016

Accepted 29 September 2016

\begin{abstract}
In order to process the linguistic-valued information with uncertainty in the financial decision- making, the present work uses a lattice-valued logical algebra - lattice implication algebra to deal with both comparable and incomparable linguistic truth-values. A new personal financial decision auxiliary modeling framework based on the lattice-ordered linguistic truth-valued logic is proposed. The concepts of linguistic-valued similarity and linguistic valued degree assignment function are introduced, and then a linguistic-valued approximate reasoning approach for financial decision making is presented. A case study is then provided which illustrates that the proposed approach is more flexible and effective with handling the financial decision-making problem involved with linguistic-valued information with uncertainty.

Keywords: Linguistic Truth-Valued Lattice Implication Algebra, Approximate Reasoning, Decision Making, Linguistic-Valued Similarity
\end{abstract}

\section{Introduction}

In the representation and processing of uncertain information ${ }^{1}$, we often get cross some information represented with linguistic values in natural language. In this regard, the researchers put forward the theories about linguistic variables ${ }^{2-3}$. People often judge the truth-values of a fuzzy proposition with linguistic hedges rather than exactly true or false, such as very true, quite false, somewhat true, slightly false and so on. A linguistic hedge possesses an intuitive meaning which can be expressed by a semantic ordering relation, e.g. Approximately True $<$ True, while Very True $>$ True. These truth values are called linguistic truth-values ${ }^{4}$.

The knowledge representation and processing are the basis of knowledge engineering and decision making. Many researchers have proposed some ideas and methods on knowledge representation. Elbedwehy et al. have proposed a semantic model (CKRMCC) based on cognitive aspects that enables cognitive computer to process the knowledge as the human mind and find a suitable representation of that knowledge ${ }^{5}$.

*corresponding author 
Bentahar et al. have proposed such a conceptual framework based on taxonomy of the most important argumentation models ${ }^{6}$. Orriols-Puig and Casillas have studied two key aspects in Fuzzy-UCS: the ability of the system to learn models from data streams where concepts change over time and the behavior of different fuzzy representations ${ }^{7}$. In intuitionistic fuzzy reasoning, $\mathrm{Xu}$ and Cai have provided a survey of the aggregation techniques of intuitionistic fuzzy information ${ }^{8}$. Many approaches have been presented for representation and reasoning of uncertain knowledge in the last decade ${ }^{9-11}$. Ha et al. have presented a similarity measure to improve the evaluation method of weighted fuzzy production rules and the multilevel fuzzy reasoning ${ }^{12}$.

The conventional linguistic-valued handling methodology is based on fuzzy set theory, which generally converts the linguistic information into a membership function, after which the generated fuzzy set will be transformed back into a word or proposition using linguistic approximation ${ }^{13}$. The process of converting each other is mainly based on the extension principle, is usually time consuming, computationally complex, and involving loss of information ${ }^{14}$. It would be more natural and reasonable to represent and reason about linguistic information in its original form, i.e., through the symbolic way ${ }^{15}$. Although most symbolic approaches have the advantages that without the loss of information and computational simplicity by avoiding use of membership function ${ }^{14,16}$, it requires that the linguistic information is totally ordered and can be manipulated by indexes. This limits the application of symbolic linguistic approach to more general situations where partially ordered information often involved.

Partially ordered information is ubiquitous in our daily decision-making problems and more flexible than total orders to represent incomplete, uncertain and imprecise knowledge ${ }^{17}$. Lattice, a special kind of partially ordered structure, has been shown to be an appropriate and efficient structure for representing ordinal qualitative information in the real world due to its additional operations and better properties ${ }^{18-19}$. Linguistic hedge algebra can be constructed as essentially a partially ordered structure according to the natural meanings of the represented linguistic terms, and generally a lattice. There have been some important works on lattice-ordered linguistic-valued based approach for decision making problems under uncertain environment ${ }^{20}$.

Furthermore, in order to establish approximate reasoning scheme with positive evidence and negative evidence, Zou and other scholars have established the linguistic truth-valued intuitionistic fuzzy lattice $\left(L I_{2 n}\right)$ which is based on the linguistic truth-valued lattice implication algebra $\left(L_{V(n \times 2)}\right)^{21-23}$. Kahramanetc et al. have surveyed the status of fuzzy multi-criteria decisionmaking methods and classified these methods into two parts: fuzzy multi-attribute decision-making (MADM) and fuzzy multi-objective decision-making (MODM) ${ }^{24}$. Feki et al. have presented a new method for ranking physical features of consumer goods according to their relevancy to multiple evaluators' perception at different levels and selecting the most important ones for quality characterization $^{25}$.

In order to process the linguistic-valued information with uncertainty in the financial decision-making, the present work proposes the algorithms of the distance and the similarity between two linguistic values based on $2 n$-element linguistic truth-valued fuzzy lattice $L_{V(n \times 2)}$, then a approximate reasoning method which can deal with both comparable and incomparable linguistic values is presented. A new personal financial decision modeling framework based on the lattice-ordered linguistic truth-valued logic is proposed. In order to grade the investors' income and deposit, a linguisticvalued degree assignment function is discussed, and a linguistic-valued approximate reasoning approach for financial decision making is presented then. An example is provided to illustrate the flexibility and effectiveness of the proposed method for handling the financial decision-making problem involved with linguisticvalued information with uncertainty.

The rest of the paper is organized as follows: Section 2 outlines some preliminary concepts about the linguistic truth-valued algebra and their corresponding propositional logic system. Section 3 presents a kind of similarity based on $2 n$-element linguistic truth-valued fuzzy lattice and establishes a linguistic-valued approximated reasoning method. A knowledge representation scheme based on linguistic-valued credibility is discussed and a financial decision-making model is constructed in Section 4. The paper is then concluded in Section 5. 


\section{Linguistic Truth-valued Propositional Logic}

\subsection{Linguistic Truth-Valued lattice implication algebra}

Definition $1 .^{23}$ Let $(L, \bigvee, \wedge O, I)$ be a bounded lattice with universal boundaries $O$ (the least element) and $I$ (the greatest element) respectively, and "'" be an orderreversing involution. For any $x, y, z \in L$, if mapping $\rightarrow: L$ $\times L \rightarrow L$ satisfies:

(1) $x \rightarrow(y \rightarrow z)=y \rightarrow(x \rightarrow z)$

(2) $x \rightarrow x=I$;

(3) $x \rightarrow y=y^{\prime} \rightarrow x^{\prime}$;

(4) $x \rightarrow y=y \rightarrow x$ implies $x=y$;

(5) $(x \rightarrow y) \rightarrow y=(y \rightarrow x) \rightarrow x$;

(6) $(x \vee y) \rightarrow z=(x \rightarrow z) \bigvee(y \rightarrow z)$;

(7) $(x \wedge y) \rightarrow z=(x \rightarrow z) \wedge(y \rightarrow z)$.

Then $\left(L, \vee, \wedge^{\prime}, \rightarrow, O, I\right)$ is a lattice implication algebra (LIA for short).

Definition 2. ${ }^{23}$ Let $A D_{n}=\left\{h_{0}, h_{1}, \ldots, h_{n}\right\}$ be a set with $n$ modifiers and $h_{0}<h_{1}<\ldots<h_{n}, M T=\{f, t\}$ be a set of meta truth values, and $f<t$. Denote $L_{V(n \times 2)}=A D_{n} \times M T$.

Define a mapping $g: L_{V(n \times 2)} \rightarrow L_{n} \times L_{2}$,

$$
g\left(\left(c_{i}, m t\right)\right)=\left\{\begin{array}{l}
\left(a_{i}, b_{1}\right), m t=f, \\
\left(a_{i}, b_{2}\right), m t=t,
\end{array}\right.
$$

Then $g$ is a bijection, denote its inverse mapping as $g^{-1}$. For any $x, y \in L_{V(n \times 2)}$, define $x \bigvee y=g^{-1}(g(x) \bigvee g(y)), x \wedge y=g^{-}$ ${ }^{1}(g(x) \wedge g(y)), x^{\prime}=g^{-1}((g(x))), x \rightarrow y=g^{-1}(g(x) \rightarrow g(y))$, then $L_{V(n \times 2)}=\left(L_{V(n \times 2)}, \vee, \wedge{ }^{\prime}, \rightarrow,\left(h_{n}, f\right),\left(h_{n}, t\right)\right)$ is called a linguistic truth-valued lattice implication algebra generated by $A D_{n}$ and $M T$.

\subsection{Six-element Linguistic Truth-Valued Proposi- tional Logic}

Definition 3. Let $H=\left\{h_{1}, h_{2}, h_{3}\right\}$ and the basic truth value set $C=\{t, f\}$, where $t=$ true, $f=$ false. Let $V$ be a linguistic truth-valued set, every linguistic truth value $v \in V$ is composed of a linguistic hedge operator and a basic word $c$, i.e. $V=H \times C$ where the linguistic hedge operator set $H$ is linear and finite set. Denote $V=\left\{\left(h_{3}, t\right)\right.$, $\left.\left(h_{2}, t\right),\left(h_{1}, t\right),\left(h_{1}, f\right),\left(h_{2}, f\right),\left(h_{3}, f\right)\right\}, L_{6}=(V, \bigvee, \wedge \rightarrow)$, the operation" id "Ared "Hasse diagram of $L_{6}$ (Fig.1), $\left(h_{i}, t\right)^{\prime}=\left(h_{i}, f\right)$ and its operations " $\rightarrow$ "are defined as follows:
(1) $\left(h_{i}, t\right) \rightarrow\left(h_{j}, f\right)=\left(h_{\max \{1, i+j-3\}}, f\right)$;

(2) $\left(h_{i}, f\right) \rightarrow\left(h_{j}, t\right)=\left(h_{\min \{3, i+j\}}, t\right)$;

(3) $\left(h_{i}, t\right) \rightarrow\left(h_{j}, t\right)=\left(h_{\min \{3,3-i+j\}}, t\right)$;

(4) $\left(h_{i}, f\right) \rightarrow\left(h_{j}, f\right)=\left(h_{\min \{3,3-j+i\}}, t\right)$.

So $L_{6}=\left(V, \vee, \Lambda^{\prime}{ }^{\prime}, \rightarrow\right)$ is a lattice implication al gebra.

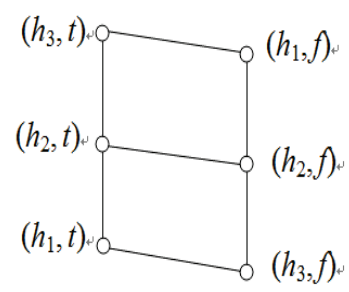

Figure1. Hasse diagram of $L_{6}$

The formal language of the six-element linguistic truth-valued propositional logic is made up of the following symbols:

1. Constant: $a, b, c, \ldots$;

2. Propositional symbol: $F, G, H, \ldots$;

3. Connective operator: $\neg, \bigvee, \wedge \rightarrow, \leftrightarrow, \ldots$;

4. Others: $($,$) .$

Definition 4. Let $P$ be an atomic proposition, then $h_{i} P$ is called an atomic proposition of $6 L T V P$, where $h_{i} \in H$.

Definition 5. The formula in $6 L T V P$ is defined as follows recursively:

(1) $6 L T V P$ atom $h_{i} P$ is a formula;

(2) If $A$ is a formula, then $\left(h_{i} A\right),(\neg A)$ is the formula, $h_{i} \in H$

(3) If $A$ and $B$ are formulas, then $(A \vee B),(A \wedge B)$, $(A \rightarrow B)$ are formulas;

(4) All the formulas are the symbolic strings which use (1) to (3) with finite times.

Definition 6. (Assignment Function) A valuation of $6 L T V P$ is a propositional algebra homomorphism, $\gamma$ : $6 L T V P \rightarrow L_{6}$.

\section{Approximate reasoning method}

\subsection{Linguistic-Valued similarity based on $L_{2 n}$}

In this section, we introduce a kind of similarity based on $2 n$-element linguistic truth-valued fuzzy lattice $L_{V(n \times 2)}$. 
Definition 7. In the $L_{V(n \times 2)}$, for any $A=\left(h_{i}, C_{k}\right), B=\left(h_{j}, C_{l}\right)$ $\in L_{V(n \times 2)}$, the distance between two linguistic values is defined as follows:

$$
d(A, B)=\left\{\begin{array}{ll}
|i-j|, & C_{k}=C_{l} \\
|i-(n-j+1)|, C_{k} \neq C_{l}
\end{array}, \text { where } 0 \leq d \leq n\right.
$$

Definition 8. In the $L_{V(n \times 2)}$, for any $A=\left(h_{i}, C_{k}\right), B=\left(h_{j}, C_{l}\right)$ $\in L_{V(n \times 2)}$, the similarity between two linguistic values is defined as follows:

$$
S(A, B)=\left\{\begin{array}{l}
\left(h_{|n-d|}, t\right), \quad C_{k}=C_{l} \\
\left(h_{(d+1)}, f\right), C_{k} \neq C_{l}
\end{array}\right.
$$

Theorem 1. In the $L_{V(n \times 2)}$, for any $A=\left(h_{i}, C_{k}\right), B=\left(h_{j}, C_{\nu}\right)$ $\in L_{V(n \times 2)}$, the following properties holds:

(1) $\left(h_{n}, f\right) \leq S(A, B) \leq\left(h_{n}, t\right)$;

(2) if and only if $A=B,\left(h_{i}, C_{k}\right)=\left(h_{j}, C_{l}\right)$ then $S(A$, $B)=\left(h_{n}, t\right)$;

(3) $S(A, B)=S(B, A)$;

(4) $S(A, B)=\left(h_{n}, f\right)$ if and only if $i=j$ and $C_{k} \neq C_{l}$;

(5) if $A \leq B \leq C$, then $S(A, C) \leq \min \{S(A, B), S(B, C)\}$.

Proof. According to Definition 7 and Definition 8

(1) When $d=0, C_{k}=C_{l}, S(A, B)$ is the largest, denote by $\left(h_{n}, t\right)$; when $d=0, C_{k} \neq C_{l}, S(A, B)$ is the smallest, denote by $\left(h_{n}, f\right)$.

(2) When $A=B$, there exists $\left(h_{i}, C_{k}\right)=\left(h_{j}, C_{l}\right)$, then $d(A$, $B)=|i-j|=0$ and $S(A, B)=\left(h_{n}, t\right)$. If $S(A, B)=\left(h_{n}, t\right)$, then $d(A, B)=|i-j|=0, C_{k}=C_{l}$. Hence $h_{i}=h_{j}$, i.e. $A=B$.

(3) We have $d(A, B)=\left\{\begin{array}{l}|i-j|, C k=C l \\ |i-(n-j+1)|, C k \neq C l\end{array}\right.$ and $d(B, A)=\left\{\begin{array}{ll}|j-i| & , C_{k}=C l \\ |j-(n-i+1)|, C k \neq C l\end{array} \quad, \quad\right.$ then $d(A$, $B)=d(B, A)$. Hence $S(A, B)=S(B, A)$ is obtained.

(4) For $\left(h_{n}, t\right)$ is the maximum element, $\left(h_{n}, f\right)$ is the minimum element in $L_{V(n \times 2)}$, so the larger the distance, the smaller the similarity.

(5) For any $A=\left(h_{i}, C_{l}\right), B=\left(h_{j}, C_{m}\right), C=\left(h_{k}, C_{n}\right) \in L_{V(n \times 2)}$. Case1: if $A \leq B \leq C$ and $C_{l}=C_{m}=C_{n}$, then $i \leq j \leq k, d(A$, $B)=|i-j|, \quad d(A, C)=|i-k|, d(B, C)=|j-k|$. Hence $d(A$, $C) \geq d(A, B), \quad d(A, \quad C) \geq d(B, \quad C)$. Because the similarity decreases when the distance increases, $S(A, C) \leq S(A, B), S(A, C) \leq S(B, C)$. So $S(A$, $C) \leq \min \{S(A, B), S(B, C)\}$.
Case2: if $A=\left(h_{i}, t\right), B=\left(h_{j}, f\right), C=\left(h_{k}, f\right)$ and $A>B>C$, $j<k$, then $d(A, B)=|i+j-n-1|, \quad d(B, C)=|j-k|, d(A$, $C)=|i+k-n-1|$. Hence $S(A, B)=\left(h_{(i+j-n-1 \mid+1)}, f\right), S(B$, $C)=\left(h_{|n-j-k-k|}, t\right), S(A, C)=\left(h_{(i+k-k n-1++1)}, f\right)$. Because $j<k$, so $|i+j-n-1|+1<|i+k-n-1|+1, \quad S(A, \quad B)=\left(h_{(i+j-n-1 \mid+1)}\right.$, f) $>S(A, C)=\left(h_{(i+k-n-1 \mid+1)}, f\right)$. So $S(A, C) \leq \min \{S(A, B)$, $S(B, C)\}$.

Case3: if $A=\left(h_{i}, t\right), B=\left(h_{j}, t\right), C=\left(h_{k}, f\right)$ and $A>B>C$, $j<i$, then $d(A, B)=|i-j|, \quad d(B, \quad C)=|j+k-n-1|, d(A$, $C)=|i+k-n-1|$. Hence $S(A, B)=\left(h_{|n-i-i j|}, \quad t\right), \quad S(B$, $C)=\left(h_{(j+k-n-1 \mid+1)}, f\right), S(A, C)=\left(h_{(|i+k-n-1|+1)}, f\right)$. Because $j<i$, so $|j+k-n-1|+1<|i+k-n-1|+1, S(B, C)=\left(h_{(j+k-n-1 \mid+1)}\right.$, f) $>S(A, C)=\left(h_{(i+k-n-1 \mid+1)}, f\right)$. So $S(A, C) \leq \min \{S(A, B)$, $S(B, C)\}$.

\subsection{Approximate reasoning method based on $L_{V(n \times 2)}$}

The approximated reasoning is simply summarized as the following steps: Firstly, we calculate the similarity between the facts and the antecedents of the rules. Then pattern matching is used to choose which rule will be activated. Secondly, by using the correction function based on the similarity measure, we construct the rule of inference phase correction after reasoning result is obtained.

Consider a simple rule, $\mathrm{R}: \quad P \rightarrow Q$. The basic reasoning model is:

Rule: $P \rightarrow Q$

Input: $P^{*}$

Output: $Q^{*}$

The algorithm of $2 n$-element linguistic-valued approximate reasoning is detailed as follows:

Step 1: Input all the rules $R_{1}, R_{2}, \ldots, R_{m}$, and set $i=1$, $j=1$.

Step 2: Input the facts $P^{*}$ (i.e. the input part of the reasoning).

Step 3: Set the threshold $\tau_{i}=\left(h_{n-i+1}, t\right)$ for the rules activation.

Step 4: Calculate the similarity $S\left(P_{j}, P^{*}\right)$ between the facts $P^{*}$ and the antecedent $P_{j}$ of the rule $R_{j}$.

Step 5: Compare $S\left(P_{j}, P^{*}\right)$ with $\tau_{i}$. If $S\left(P_{j}, P^{*}\right)=\tau$, then the corresponding rule $R_{j}$ will be activated and go to Step 6. If $j<m$, then set $j=j+1$ and go to Step 4. If $i<3$, then set $i=i+1$ and go to Step 3 .

Step 6: Take $S\left(Q_{j}, Q^{*}\right)=S\left(P_{j}, P^{*}\right)$, where $Q_{j}$ is the after of the rule $R_{j}$. Then we can obtain the approximate reasoning results $Q^{*}$ through formula (1) and (2). 
Step 7: Output the conclusion $Q^{*}$ and terminate the algorithm.

Note 1: It is worth mentioning that the selection of the threshold $\tau$ is closely related to the application field, so the system actual case study is required before operation. If there is an unsatisfactory result, one can choose different threshold values.

\section{Linguistic-Valued Approximate Reasoning Model for Financial Decision Making}

\subsection{Knowledge representation model based on $L_{6}$}

The knowledge representation method proposed in this paper is on the basis of propositional logic representation. We express the values of knowledge by linguistic values directly.

The following is an example of a simple financial investment decision making problem. The function of the auxiliary decision-making process is to help users decide whether save their money or invest in the stock market. Some investors may put their money on these two aspects. Individual investors' investment scheme depends on their income and the bank deposit, according to the following principle:

(1) if the investor has less deposit, then all of his income should be saved;

(2) if the investor has much more deposit and income, then he could consider investing in stocks;

(3) if the investor has more deposits and income, then he should invest most of the extra money to buy stocks and save the few;

(4) if the investor has less deposit and his income is much more, then he should save most of the extra money and invest the few to buy stocks.

Note that if the investor is low-income or very little income, then he is regarded as has no extra money.

Here the capital letters represent the investors. The lowercase letters represent the functions. Taking the individual as the universe, requirements:

- $D$ represents investor has deposit;

- I represents investor has income;

- IS represents that the investor should invest in stocks;

- $\quad I M$ represents that the investor should save money;

- $M S$ represents that the money for the stock is greater than the money saved in the bank;
- $\quad M M$ represents that the money saved in the bank is greater than the money for the stock.

Assume that

1. The value of investor's much more deposits is: $\left(h_{3}\right.$, t) $D$;

2. The value of investor's more deposits is: $\left(h_{2}, t\right) D$;

3. The value of investor's less deposits is: $\left(h_{1}, t\right) D$;

4. The value of investor's much more income is: $\left(h_{3}\right.$, t)I ;

5. The value of investor's more income is: $\left(h_{2}, t\right) I$;

6. The value of investor's less income is: $\left(h_{1}, t\right) I$;

The different investment strategies are represented by a logical expression."

- Rule 1 can be expressed as $\left(h_{1}, t\right) D \rightarrow I M$.

- $\quad$ Rule 2 can be expressed as $\left(h_{3}, t\right) D \wedge\left(h_{3}, t\right) I \rightarrow I S$.

- Rule 3 can be expressed as $\left(h_{2}, t\right) D \wedge\left(h_{2}, t\right)$ $I \rightarrow I S$ NM AMS.

- Rule 4 can be expressed as $\left(h_{1}, t\right) D \wedge\left(h_{3}, t\right)$ $I \rightarrow I S \wedge M \wedge M M$.

\subsection{Financial decision-making model}

Considering the specific linguistic-valued data, if we want to help the investors to make rational investment, firstly, we need to grade his income and deposit, then according to the different degrees of income and deposit to provide him with different advices. The income and deposit of the investors can be divided into three degrees based on $L_{6}$. We denote the highest income and deposit as $\alpha_{T}$ and the lowest income and deposit as $\alpha_{F}$ in the whole data. The $\varepsilon_{T}$ is the highest floating value and the $\varepsilon_{F}$ is the lowest floating value. So naturally if an investor's income is more than the difference between the highest income and the highest floating value, then his income is much more; if an investor's income is less than the difference between the lowest income and the lowest floating value, then his income is less; otherwise his income is more. Similarly, we can get the degree of the investor's deposit. The definition of the linguisticvalued hierarchical assignment function is as follows.

Definition 9 For any survey data set $X, \forall x \in X, V_{(x)}$ is linguistic-valued hierarchical assignment function (Fig.2), expressed as

$$
V(x)=\left\{\begin{array}{l}
(h 1, t), x \leq \alpha_{F}+\varepsilon_{F} \\
(h 2, t), \alpha_{F}+\varepsilon_{F}<x<\alpha_{T}-\varepsilon_{T} \\
(h 3, t), x \geq \alpha_{T}-\varepsilon_{T}
\end{array}\right.
$$


Table1. Income and savings survey

\begin{tabular}{ccccc}
\hline area & $\begin{array}{c}\text { High income } \\
( \pm \$ 500)\end{array}$ & $\begin{array}{c}\text { Low income } \\
( \pm \$ 100)\end{array}$ & $\begin{array}{c}\text { More deposit } \\
\text { (ten thousand) }( \pm \$ 2)\end{array}$ & $\begin{array}{c}\text { Less deposit } \\
\text { (ten thousand) }( \pm \$ 1)\end{array}$ \\
\hline Alaska & 11000 & 1340 & 16.0 & 8.2 \\
Florida & 14400 & 2000 & 21.0 & 10.0 \\
Colorado & 5000 & 920 & 10.0 & 5.6 \\
Alabama & 7000 & 1100 & 12.4 & 6.8 \\
\hline
\end{tabular}

where $\alpha_{T}$ as the highest one and $\alpha_{F}$ as the lowest one in $X$. The $\varepsilon_{T}$ is the highest floating value and the $\varepsilon_{F}$ is the lowest floating value.

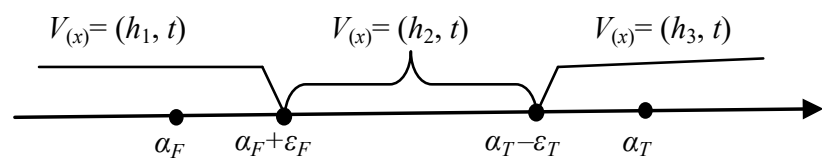

Figure2. Linguistic-valued hierarchical assignment function based on $L_{6}$.

Example 1 In Table 1, as to the high income concept, Florida comprehensive data corresponding to the highest is $14400 \$$, so $\alpha_{T}=14400 \$, 500 \$$ for its elastic range $\varepsilon_{T}$; for the low-income concept, low comprehensive survey data of Colorado is $920 \$$, so $\alpha_{F}=920, \varepsilon_{F}=100$ for its elastic range. Therefore, for the income:

$$
V(x)=\left\{\begin{array}{l}
\left(h_{1}, t\right), x \leq 1020 \\
\left(h_{2}, t\right), 1020<x<13900 \\
\left(h_{3}, t\right), x \geq 13900
\end{array}\right.
$$

The same for deposit:

$$
V(x)=\left\{\begin{array}{l}
\left(h_{1}, t\right), x \leq 6.6 \\
\left(h_{2}, t\right), 6.6<x<19 \\
\left(h_{3}, t\right), x \geq 19
\end{array}\right.
$$

According to the above analysis, the financial investigation decision-making algorithm based on sixelement linguistic-valued approximate reasoning approach is as follows. $j=1$.
Step 2: Calculate the linguistic-valued evaluation $\left(h_{k}, t\right)$ of the user's income and deposit through the linguistic-valued hierarchical assignment function.

Step 3: Set the threshold $\tau_{i}=\left(h_{n-i+1}, t\right)$ for the rules activation.

Step 4: Calculate the similarity $S_{x}$ between $\left(h_{k}, t\right)$ and the value of the antecedent $\left(h_{l}, t\right)$ of the rule $R_{j}$.

Step 5: Compare $S_{x}$ with $\tau$. If $S_{x}=\tau_{i}$, then the corresponding rule $R_{j}$ will be activated and go to Step 6 . If $j<m$, then set $j=j+1$ and go to Step 4 . If $i<3$, then set $i=i+1$ and go to Step 3 .

Step 6: Execute the rule $R_{j}$ and output the conclusion.

\subsection{Example Illustration}

Example 2 Suppose there is one person lives in Alaska, a monthly salary is $\$ 5000$, household deposits is $\$ 120000$. He wants to know how to make investment programs. According to linguistic-valued hierarchical assignment function, the corresponding degree of the rules can be obtained as follows.

(1) $V_{(D(x))}=V_{(D(12))}=\left(h_{2}, t\right)$;

(2) $V_{(I(x))}=V_{(I(5000))}=\left(h_{2}, t\right)$;

(3) $V_{(D(12)))} \wedge V_{(I(5000))}=\left(h_{2}, t\right)$.

Firstly let $\tau_{1}=\left(h_{3}, t\right)$, for the distance $d$ of the facts and the antecedent of the rules is 0 , then the facts and the antecedent of the rules are extremely similar. According to Definition 7 and Definition 8,

(1) Rule 1: $\left(h_{1}, t\right) D \rightarrow I M$.

The matching degree of the precondition $S_{1}=\left(h_{|3-1|}, t\right)<\tau_{1}$ because $d_{1}=1$, so the Rule 1 can't be executed.

(2) Rule 2: $\left(h_{3}, t\right) D \wedge\left(h_{3}, t\right) I \rightarrow I S$.

The matching degree of the precondition $S_{2}=\left(h_{|3-1|}, t\right)<\tau_{1}$ because $d_{2}=1$, so the Rule 2 can't be executed.

(3) Rule 3: $\left(h_{2}, t\right) D \wedge\left(h_{2}, t\right) I \rightarrow I S \wedge M \wedge M S$.

The matching degree of the antecedent $S_{3}=\left(h_{|3-0|}, t\right)=\tau_{1}$ because $d_{3}=0$, so it is executed.

(4) Rule 4: $\left(h_{1}, t\right) D \wedge\left(h_{3}, t\right) I \rightarrow I S \wedge M \wedge M M$. 
The matching degree of the precondition $S_{4}=\left(h_{|3-1|}, t\right)<\tau_{1}$ because $d_{4}=1$, so the Rule 4 can't be executed.

In conclusion, the person can adopt Rule 3, i.e. he should use most of the extra money to buy stocks and save the few. If the threshold $\tau_{1}=\left(h_{3}, t\right)$ can't activate any rules then let $\tau_{2}=\left(h_{2}, t\right)$ or $\tau_{3}=\left(h_{1}, t\right)$ until one rule can be activated.

In general, we use linguistic truth-valued propositional logic to express human's language information effectively. The financial decision making system based on approximate reasoning method in $L_{6}$ makes knowledge representation more comprehensive and the decision results are more effective with linguistic values.

\section{Conclusions}

This paper proposed an approximate reasoning approach based on linguistic truth-valued lattice implication algebra for financial decision-making problem with the linguistic information. Knowledge representation with linguistic values has a very wide range of application prospects. Based on the linguistic truth-valued propositional logic, we present an extended knowledge representation method and apply it to financial decision making. The proposed approach can deal with both comparable and incomparable linguistic-valued information under uncertain circumstances. Further work is to use the linguistic-valued approximate reasoning approach into the big data analytics. It is an important research that how to discover the rules and get a reasonable results with linguistic-valued data in some fields such as risk analysis, decision-making and so on.

\section{Acknowledgements}

This work is partially supported by National Natural Science Foundation of China (No. 61672127) and National Natural Science Foundation of Liaoning Province (Nos. 2015020059 and 201602464).

\section{References}

1. L. A. Zadeh.: Toward a generalized theory of uncertainty (GTU)-an outline [J]. Information Sciences, 172:1-40(2005).
2. N.C. Ho, A topological completion of refined hedge algebras and a model of fuzziness of linguistic terms and hedges, Fuzzy Sets and Systems. 158: 436-451(2007).

3. N.C. Ho and N. V. Long, Fuzziness measure on complete hedge algebras and quantifying semantics of terms in linear hedge algebras, Fuzzy Sets and Systems. 158: 452-471(2007).

4. L. Zou, D. Ruan, Z. Pei and Y. Xu, A linguistic truthvalued reasoning approach in decision making with incomparable information, Journal of Intelligent and Fuzzy Systems, 19(4-5): 335-343(2008).

5. M. N. Elbedwehy, M. E. Ghoneim, A. E. Hassanien and A. T. Azar, A computational knowledge representation model for cognitive computers, Neural Computing \& Applicication, 25:1517-1534(2014).

6. J. Bentahar, B. Moulin and M. Bélanger, A taxonomy of argumentation models used for knowledge representation, ArtifIntell Rev, 33:211-259(2010).

7. A. Orriols-Puig and J. Casillas, Fuzzy knowledge representation study for incremental learning in data streams and classification problems, Soft Computing, 15:2389-2414(2011).

8. Z. $\mathrm{Xu}$ and $\mathrm{X}$. Cai, Recent advances in intuitionistic fuzzy information aggregation, Fuzzy Optimize Decision Making, 9:359-381(2010).

9. Z. Pei, Y. Xu, D. Ruan and K. Qin, Extracting complex linguistic data summaries from personnel database via simple linguistic aggregations, Information Sciences. 179: 2325-2332(2009).

10. L. Zou, S. Fang and Y. Xu.: Resolution method of sixelement linguistic truth-valued intuitionistic propositional logic $[\mathrm{J}]$, in Proc. 3rd International Conference on Intelligent System and Knowledge Engineering.141-145(2008).

11. X.M. Zhong, Y. Xu, J. Liu, D. Ruan and S.W. Chen General form of $\alpha$-resolution based on linguistic truthvalued lattice-valued logic $[\mathrm{J}]$. Soft Computing, 10:1767-1781(2012).

12. M.-H. Ha, Y. Li and X.-F. Wang, Fuzzy knowledge representation and reasoning using a generalized fuzzy petri net and a similarity measure, Soft Computing, 11: 323-327(2007).

13. J.M. Mendel, Computing with words and its relationships with fuzzistics, Information Sciences 177 (4) (2007) 988-1006.

14. F. Herrera and L. Martínez, The 2-tuple linguistic computational model: advantages of its linguistic description, accuracy and consistency, International Journal of Uncertainty, Fuzziness and Knowledge-based Systems 9 (2001) 33-48.

15. Y. Xu, J. Liu, L. Martínez and D. Ruan, Some views on information fusion and logic based approaches in decision making under uncertainty, Journal of Universal Computer Science 16 (1) (2010) 3-21. 
16. L. Martínez and F. Herrera, An overview on the 2-tuple linguistic model for computing with words in decision making: extensions, applications and challenges, Information Sciences 207 (2012) 1-18.

17. B. Malakooti, Ranking and screening multiple criteria alternatives with partial information and use of ordinal and cardinal strength of preferences, IEEE Transactions on Systems, Man, and Cybernetics-Part A 30 (3) (2000) 355-368.

18. G. Birkhoff, Lattice Theory, 3rd ed., American Mathematical Society, Providence, RL, 1967.

19. V.G. Kaburlasos, G.X. Ritter, Computational Intelligence Based on Lattice Theory, Springer, Heidelberg, 2007.

20. Z. Pei, D. Ruan, J. Liu, and Y. Xu, Linguistic Values Based Intelligent Information Processing: Theory, Methods, and Applications, Atlantis Press/World Scientific, Paris/Singapore, December, 2009.

21. L. Zou, Z. Pei, X. Liu and Y. Xu, Semantics of linguistic truth-valued intuitionistic fuzzy proposition calculus,
International Journal of Innovative Computing, Information and Control. 5(12): 4745-4752(2009).

22. L. Zou, X. Liu, Z. Wu and Y. Xu, A uniform approach of linguistic truth values in sensor evaluation, International Journal of Fuzzy Optimization and Decision Making. 7(4): 387-397(2008).

23. L. Zou, D. Ruan, Z. Pei and Y. Xu. A linguistic-valued lattice implication algebra approach for risk analysis, Journal of Multi-Valued Logic and Soft Computing. 17(4):293-303 (2011).

24. C. Kahramana, S. C. Onara and B. Oztaysia., Fuzzy Multicriteria Decision-Making: A Literature Review [J].International Journal of Computational Intelligence Systems, 8(4):637-666(2015)

25. Fekia, X. Fengb, A. Ghithc, L. Koehlb, F. Msahlia and F. Saklid., Selecting Multiple Evaluator's PerceptionOriented Relevant Physical Features of Consumer Goods by Using Fuzzy Data Sensitivity and OWA Operators[J].International Journal of Computational Intelligence Systems, 9(2):213-226(2016). 